\title{
Opioid prescriptions are associated with hepatic encephalopathy in a national cohort of patients with compensated cirrhosis
}

\author{
Andrew M. Moon ${ }^{1}$ (D) | Yue Jiang ${ }^{2}$ | Shari S. Rogal ${ }^{3}$ | Elliot B. Tapper ${ }^{4,5}$ (D) | \\ Sarah R. Lieber ${ }^{1}$ | A. Sidney Barritt IV ${ }^{1}$
}

${ }^{1}$ Division of Gastroenterology and Hepatology, University of North Carolina at Chapel Hill, Chapel Hill, NC, USA

${ }^{2}$ Department of Biostatistics, University of North Carolina Gillings School of Global Public Health, Chapel Hill, NC, USA

${ }^{3}$ Division of Gastroenterology, Hepatology, and Nutrition, University of Pittsburgh, Pittsburgh, PA, USA

${ }^{4}$ Division of Gastroenterology and Hepatology, University of Michigan, Ann Arbor, MI, USA

${ }^{5}$ Gastroenterology Section, VA Ann Arbor Healthcare System, Ann Arbor, MI, USA

\section{Correspondence}

Dr Andrew M. Moon, 130 Mason Farm Road, Bioinformatics Building CB\# 7080, Chapel Hill, NC 27599-7080, USA.

Email: andrew.moon@unchealth.unc.edu

Funding information

This research was supported in part by NIH grant T32 DK007634 (A.M. and S.L.).

\section{Summary}

Background: Opioids are often prescribed for pain in cirrhosis and may increase the risk of hepatic encephalopathy (HE).

Aim: To assess the association between opioids and HE in patients with well-compensated cirrhosis.

Methods: We used the IQVIA PharMetrics (Durham, NC) database to identify patients aged 18-64 years with cirrhosis. We excluded patients with any decompensation event from 1 year before cirrhosis diagnosis to 6 months after cirrhosis diagnosis. Over the 6 months after cirrhosis diagnosis, we determined the duration of continuous opioid use and classified use into short term (1-89 days) and chronic (90180 days). We assessed whether patients developed HE over the subsequent year (ie 6-18 months after cirrhosis diagnosis). We used a landmark analysis and performed multivariable Cox proportional hazards regression to assess associations between opioid use and $\mathrm{HE}$, adjusting for relevant confounders.

Results: The cohort included 6451 patients with compensated cirrhosis, of whom $23.3 \%$ and $4.7 \%$ had short-term and chronic opioid prescriptions respectively. Over the subsequent year, $\mathrm{HE}$ occurred in $6.3 \%$ patients with chronic opioid prescriptions, $5.0 \%$ with short-term opioid prescriptions and $3.3 \%$ with no opioid prescriptions. In the multivariable model, an increased risk of HE was observed with short-term (adjusted hazard ratio, HR $1.44,95 \% \mathrm{Cl} 1.07-1.94$ ) and chronic opioid prescriptions (adjusted HR 1.83, 95\% Cl 1.07-3.12) compared to no opioid prescriptions.

Conclusion: In this national cohort of privately insured patients with cirrhosis, opioid prescriptions were associated with the risk of incident HE. Opioid use should be minimised in those with cirrhosis and, when required, limited to short duration. 


\section{1 | INTRODUCTION}

Hepatic encephalopathy (HE) is a debilitating and common complication of cirrhosis. ${ }^{1} \mathrm{HE}$ is independently associated with decreased health-related quality of life and reduced survival. ${ }^{2,3}$ Sedating and psychoactive medications such as opioids may contribute to the risk of $\mathrm{HE}$ through multiple mechanisms. For example, opioids could lead to altered gut microbiota, upregulation in central mu-opioid receptors and slowed intestinal motility, which could increase intestinal absorption of ammonia and inflammatory mediators. ${ }^{4-7}$ Despite their potential harms, opioid use is frequent and increasing over time in patients with cirrhosis, possibly due to the avoidance of nonsteroidal anti-inflammatory drugs and acetaminophen in these patients. ${ }^{8-13}$ In an era of increasing opioid use, the relative risk of $\mathrm{HE}$ among patients taking opioids remains unclear. $^{8}$

Studies examining the association between opioids and $\mathrm{HE}$ have been conflicting and there have been no studies demonstrating an association between opioids and $\mathrm{HE}$ in younger patients with well-compensated cirrhosis. A study within the United States Medicare population demonstrated that opioid prescriptions were associated with increased incidence of $\mathrm{HE} .{ }^{14}$ However, given the data source, this population was older and may have been more susceptible to centrally acting medications due to decreased baseline cognitive reserve. It may be that younger patients with cirrhosis are able to safely tolerate opioids. In fact, one single-centre study reported that an opioid prescription at discharge increased overall readmissions, but not hepatic encephalopathy-related readmissions over 6 months. ${ }^{6}$ Furthermore, in a longitudinal cohort of United States veterans, baseline opioid use was not found to be an independent predictor of hepatic encephalopathy. ${ }^{15}$

Therefore, given the uncertain association between opioids and $\mathrm{HE}$, we aimed to assess the association between opioid use and incident hepatic encephalopathy among a nationally representative cohort of younger, privately insured patients with no prior decompensation events. We also aimed to assess whether duration of opioid prescription influences the risk of $\mathrm{HE}$. We hypothesised that opioid prescriptions and particularly chronic use would be associated with incident $\mathrm{HE}$.

\section{2 | MATERIALS AND METHODS}

\subsection{Data source}

Our data source was the IQVIA Pharmetrics (Durham, NC) database from 1 January 2007 to 30 September 2015. This database includes claims data on pharmacy and medical coverage for more than 27 million Americans with private health insurance. This data source has been used by others and has been demonstrated to be demographically representative of commercially insured US patients. ${ }^{16}$
Given that data were obtained from a de-identified database, the University of North Carolina Institutional Review Board deemed this project to be exempt from full review.

\section{2 | Patient population}

We identified all patients aged 18-64 years with cirrhosis, defined as the presence of two or more International Classification of Diseases, Ninth Revision (ICD-9) codes for cirrhosis (571.5 or 571.2) on separate dates. Previous validation studies have demonstrated that the presence of two outpatient ICD-9 codes improves the positive predictive value of correctly identifying cirrhosis based on a gold standard of medical record review. ${ }^{17,18}$ The 'diagnosis date' of cirrhosis was defined as the first ICD-9 code present in the database.

We limited our analysis to patients without prior decompensation events, allowing us to capture truly incident $\mathrm{HE}$ events in a population with newly identified cirrhosis. In order to define this well-compensated population, we excluded patients with any ICD-9 codes, Current Procedural Terminology codes or pharmacy claims for $\mathrm{HE}$, varices/variceal haemorrhage, spontaneous bacterial peritonitis, ascites, hepatorenal syndrome/end-stage renal disease, hepatocellular carcinoma, transjugular intrahepatic portosystemic shunt procedure or jaundice (Table S1). We also excluded patients who underwent prior liver transplantation.

We required at least 12 months of continuous health plan enrolment with pharmacy benefits before the diagnosis of cirrhosis. This run-in period was used to determine patient demographics, comorbidities, aetiology of cirrhosis (alcohol-related liver disease [ALD], viral hepatitis, other), complications of liver disease and other medications (Tables S1 and S2). Additionally, we required 18 months of continuous health plan enrolment with pharmacy benefits after the diagnosis of cirrhosis. We limited enrolment to those $\leq 64$ years old to avoid patients with dual Medicare enrolment whose claims may not be captured accurately in this database.

\section{3 | Exposure assessment}

Our exposure was defined as a claim for a prescription opioid in the outpatient setting over the 6 months after cirrhosis diagnosis. The 6-month exposure period was chosen a priori to provide sufficient power to assess the association between opioid prescriptions and $\mathrm{HE}$ and to reduce the risk of confounding by indication and misclassification of opioid use. We performed sensitivity analyses using exposure periods of 3 and 9 months.

The primary analysis included all opioids listed in Table S2 and a sensitivity analysis excluded tramadol and propoxyphene. We categorised opioid prescriptions as short term (1-89 days) and chronic ( $\geq 90$ days) based on the longest continuous opioid prescription recorded, allowing gaps of up to 7 days. Additional information on the assessment of exposures is shown in Figure 1. 


\section{4 | Outcome assessment}

Our primary outcome was time to incident hepatic encephalopathy occurring from 6 to 18 months after cirrhosis diagnosis. We defined $\mathrm{HE}$ as the presence of at least one ICD-9 code (572.2) and/or prescription for lactulose or rifaximin. We used a combination of ICD codes and medications given the low negative predictive value of ICD codes alone for identifying HE episodes. ${ }^{19,20}$

We performed a secondary analysis examining the association between opioid prescriptions and any episode of non-HE decompensation event (composite outcome): variceal haemorrhage, ascites, spontaneous bacterial peritonitis, hepatorenal syndrome or placement of transjugular intrahepatic portosystemic shunt (presumably for variceal haemorrhage or ascites) (Table S1).

\section{5 | Sensitivity analyses}

We performed several sensitivity analyses to assess internal validity of the database. We first assessed the association between positive and negative control medications and HE. Positive controls (ie active comparators) are medications that may be used to treat pain and thus evaluate for unmeasured confounders common among those with pain. ${ }^{21,22}$ Our positive controls were tricyclic antidepressants and gabapentin. Negative controls are medications that are not prescribed for pain and unlikely to cause the outcome of interest. Our negative controls, which included statins and levothyroxine, can assess for healthy user bias or confounding by selective prescribing.
These biases result due to preferential prescribing of medications for patients who are healthier at baseline and avoidance of prescribing among those who are frail or particularly ill. Lastly, we assessed benzodiazepine use given that these medications are often used in combination with opioids and may confound the relationship between opioids and HE. In these analyses, we examined the adjusted hazard ratios (HRs) for models where an additional category of 'control drug only' (eg gabapentin, tricyclic antidepressants, levothyroxine, statins, benzodiazepines) was added to the exposure definition.

Additionally, we performed a sensitivity analysis employing a true new user design, in which we excluded patients with an opioid prescription within the 6 months prior to cirrhosis diagnosis.

\section{6 | Statistical analysis}

We assessed baseline characteristics among those with no opioid prescriptions, short-term prescriptions and chronic opioids prescriptions. For continuous variables, we reported medians with interquartile ranges and reported proportions for categorical variables. We performed bivariate comparisons by opioid exposure using KruskalWallis for continuous variables and chi-squared for categorical variables. Statistical analyses were carried out using R version 3.4.2.

We used a landmark analysis that aims to estimate the time-toevent probabilities in each exposure category, conditional on the exposure status of patients at the landmark time (Figure 1). ${ }^{23}$ In a landmark analysis, the point at which we evaluate the exposure status of patients is fixed and the analysis is limited to patients who have

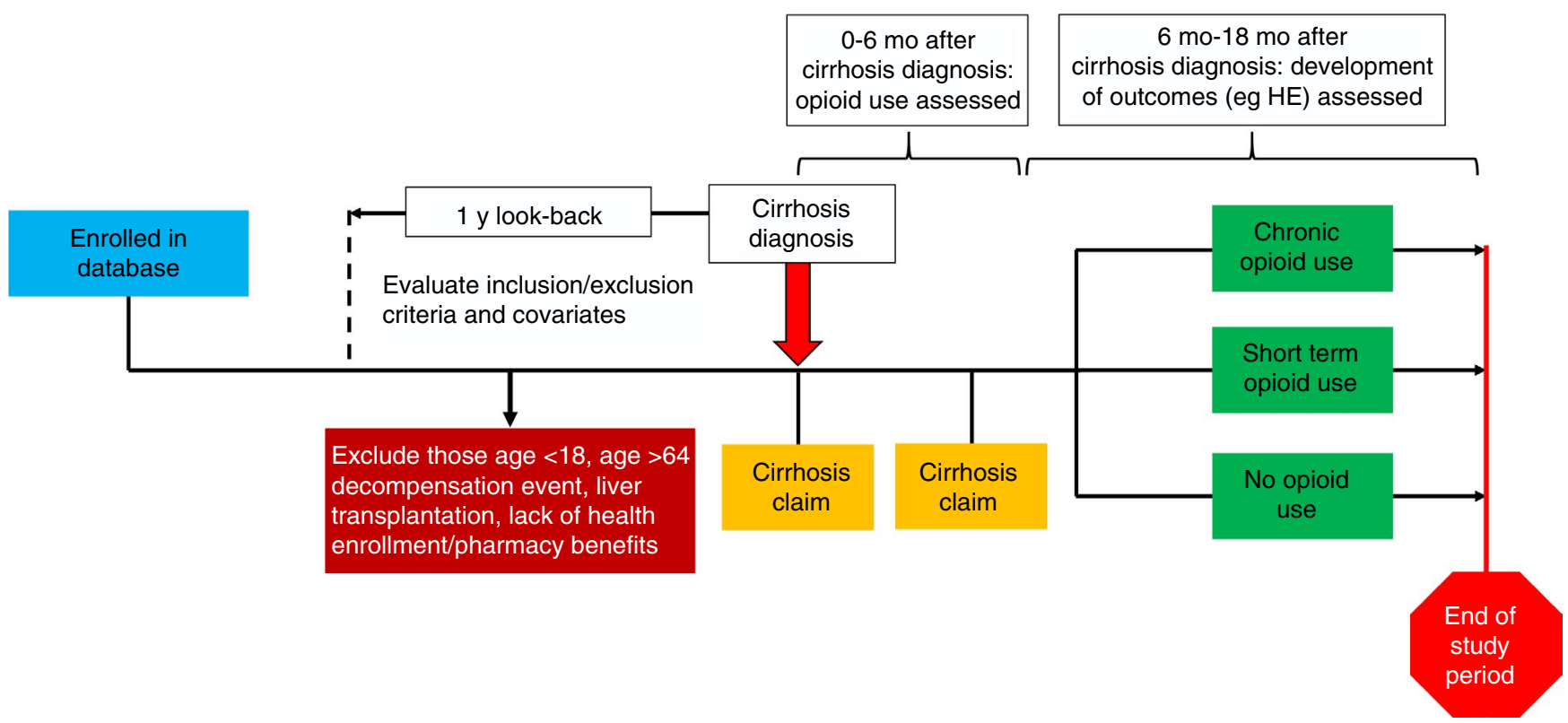

FIGURE 1 Overview of assessment of exposure to opioids and outcomes including HE and non-HE decompensation events. All patients enrolled in the IQVIA Pharmetrics database with at least two ICD-9 codes for cirrhosis were included in the cohort. There was a 1-y lookback period prior to cirrhosis diagnosis during which covariates and exclusion criteria were assessed. Patients aged 18-64 y with continuous health enrolment/pharmacy benefits and no decompensation events were included. Exposure to opioids was assessed in the 6 mo after cirrhosis diagnosis. In the year after the exposure assessment period (ie 6 mo-1 y after cirrhosis diagnosis), patients were assessed for the presence of $\mathrm{HE}$ or non-HE decompensation events 
not yet experienced our outcome of interest (HE) by the landmark time, which was set at 6 months after cirrhosis diagnosis. We thus exclude all patients who experience $\mathrm{HE}$ before the landmark time and ignore all opioid claims after the landmark time. As a result, this analysis reduces the risk of bias resulting from a patient's opioid classification changing through the course of the follow-up time and ensures a uniform exposure assessment for patients who do and do not develop $\mathrm{HE}$. In addition, this approach limits confounding by indication that may occur if patients receive opioids for an event that increases their likelihood of HE, such as a surgical procedure. Furthermore, this approach limits inclusion of patients prescribed lactulose for opioid-induced constipation or prescribed opioids and, as a result, given lactulose or rifaximin for primary prophylaxis against HE. Lastly, we chose to censor follow-up data at 1-year after the landmark time, since HE events that occur $>1$ year after an opioid prescription are unlikely to be related to this prescription.

We used multivariable Cox proportional hazards regression to assess associations between opioid use and development of $\mathrm{HE}$, adjusting for age, sex, region, cirrhosis aetiology, depression, Charlson comorbidity index $(\mathrm{CCl})$ and prior opioid use. We also performed multivariable Cox proportional hazards regression to assess the association between opioid use and non-HE decompensation events. These covariates were chosen a priori based on their potential association with both opioid use and development of $\mathrm{HE}$.

\section{3 | RESULTS}

\section{1 | Baseline characteristics and opioid use}

The cohort included 6451 patients without any decompensation events within 1 year prior to cirrhosis diagnosis (Figure 2). The median age at cirrhosis diagnosis was 54 years $\left(\mathrm{p}_{25}-\mathrm{p}_{75} 49-58\right)$ and $57.9 \%$ were male. Other baseline patient characteristics can be found in Table 1.

Overall, 1806 patients (28.0\%) were prescribed opioids during the 6 months after cirrhosis diagnosis. Among this group, the median cumulative days of opioids prescribed was 8 days $\left(p_{25}-p_{75} 2-40\right)$. Shortterm opioids were used by 1505 patients (23.3\%) and chronic opioids were used by 301 patients (4.7\%). The median duration of use was 5 and 153 days among short-term and chronic opioid prescriptions respectively. The most common opioids used were oxycodone, tramadol and fentanyl. Those with short-term and chronic opioid prescriptions had a higher proportion of female patients, non-ALD aetiology, $\mathrm{CCl}>2$, diabetes mellitus and depression (Table 1).

\section{2 | Development of hepatic encephalopathy}

Over a 1-year follow-up period, 250 (3.9\%) of the cohort developed HE (Table 2). HE incidence was highest among those with chronic opioid prescriptions (6.3\%) compared to short-term opioids $(5.0 \%)$ and no opioids (3.3\%) (Figure 3). In the multivariable Cox proportional hazards model, both short-term opioid prescriptions (adjusted
HR 1.44, 95\% Cl 1.07-1.94) and chronic opioid prescriptions (adjusted HR 1.83, 95\% Cl 1.07-3.12) were significantly associated with the risk of hepatic encephalopathy.

For our sensitivity analysis using a 3-month exposure period, the rates of $\mathrm{HE}$ between 3 and 18 months after index remained higher among opioid users (5.3\%) compared to non-opioid users (4.9\%) but this difference was not statistically significant in the multivariable model (adjusted HR 1.17, 95\% Cl 0.96-1.44). When using a 9-month exposure period, HE rates from 9 to 21 months after index were lowest among those without opioid prescriptions ( $n=120,3.0 \%$ ) compared to those with short-term ( $\mathrm{n}=66,5.2 \%$ ) and chronic prescriptions ( $n=16,5.1 \%$ ). In the multivariable Cox proportional hazards models, opioid use was significantly associated with $\mathrm{HE}$ among short-term users (AHR 1.73, 95\% $\mathrm{Cl}$ 1.26-2.33) but not significantly associated among chronic users (AHR 1.54, 95\% Cl 0.912.62), although the direction of the association remained. Lastly, in the sensitivity analysis excluding tramadol and propoxyphene from the exposure definition there remained a statistically significant association between opioid use and HE among paitents with both short-term opioids (adjusted HR 1.88, 95\% Cl 1.43-2.46) and chronic opioids (adjusted HR 2.34, 95\% Cl 1.59-3.44).

In all sensitivity analyses of positive and negative controls, both levels of opioid use remained significantly associated with $\mathrm{HE}$, while prescriptions for gabapentin only (adjusted HR 1.68, 95\% Cl 0.624.59), tricyclic antidepressants only (adjusted HR 0.90, 95\% Cl 0.441.86), levothyroxine only (adjusted HR $1.34,95 \% \mathrm{Cl} 0.49-3.64$ ) and statins only (adjusted HR $0.74,95 \% \mathrm{Cl} 0.39-1.41$ ) were not significantly associated with the development of HE. Benzodiazepines only were associated with a significantly increased risk of $\mathrm{HE}$ in the unadjusted analysis (HR 1.77, 95\% Cl 1.17-2.68) but the association was not statistically significant in the multivariable model (adjusted HR 1.51, 95\% Cl 0.98-2.31).

When excluding all patients with an opioid prescription within 6 months prior to cirrhosis diagnosis, the associations remained directionally similar and significant for short-term opioid use (adjusted HR 1.47, 95\% Cl 1.03-2.09) but was no longer statistically significant for chronic opioid use (adjusted HR 1.55, 95\% Cl 0.38-6.30).

There were no missing data for the unadjusted models examining the association between opioid prescriptions and HE. For adjusted models, there were 306 missing observations (4.7\%) for our primary analysis and all subgroup analyses. Individuals with missing data were excluded from these models.

\subsection{Other decompensation events}

Overall, 771 patients (12.0\%) also developed non-HE decompensation events over 1 year, with similar rates among those with no opioid prescriptions (12.0\%), short-term opioids (12.0\%) and chronic opioids (11.6\%) (Figure 4). These non-HE decompensation events included 718 with ascites, 385 with variceal haemorrhage, 13 with spontaneous bacterial peritonitis and eight with hepatorenal syndrome. In the multivariable Cox proportional hazards models, neither 
Excluded:

- 27659333 patients without $\geq 2$ ICD-9 codes for cirrhosis

- 46969 patients without $\geq 12$ mo of

continuous enrolment with pharmacy benefits

- 36766 with <2 cirrhosis codes b/w age 18-64

- 22402 patients without $\geq 12$ mo of look-

back time prior to cirrhosis diagnosis

- 19020 patients with a decompensation event in

the 12 mo look-back period

- 4240 without $18 \mathrm{mo}$ of follow-up after

cirrhosis index date or with HE within 6 mos of

index date

- 3587 who had a liver transplant code

- 1255 patients who only had PBC codes for

cirrhosis

27800023 commercially-

insured patients within IMS

database



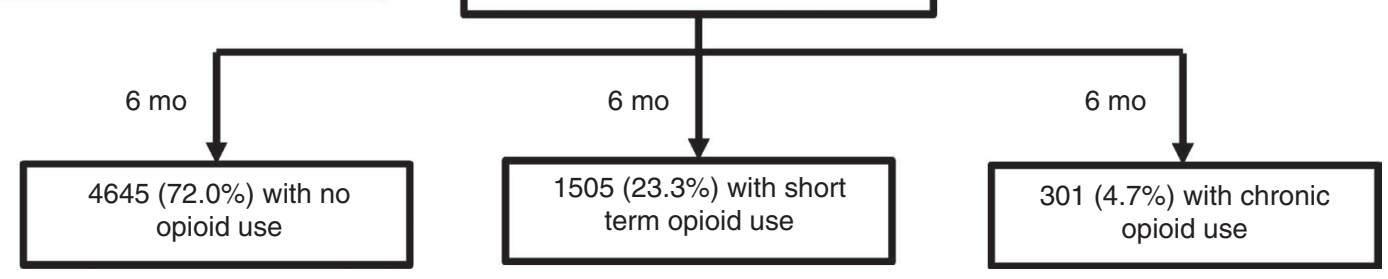

FIGURE 2 Flow diagram of cirrhosis patients including in our analysis cohort. The cohort included patients with at least two ICD-9 codes for cirrhosis with sufficient insurance/pharmacy coverage, a 12-mo look-back, no decompensation events during the look-back and no episodes of HE during the 6 mo after cirrhosis index date. Our final cohort included 6451 well-compensated cirrhosis patients who were followed for 6 mo to assess their exposure to opioids and then for a further 1 yr to assess for the development of incident $\mathrm{HE}$

short-term opioids (adjusted HR 0.96, 95\% Cl 0.80-1.16) nor chronic opioids (adjusted HR 0.93, 95\% Cl 0.64-1.35) were associated with a statistically significantly increased risk of non-HE decompensation events.

\section{4 | DISCUSSION}

In this nationwide cohort of privately insured patients with cirrhosis, both short-term and chronic opioid prescriptions were independently associated with an increased risk of HE. Our study provides several novel findings. First, $28 \%$ of cirrhosis patients received opioids and that the annual incidence of HE development was 1.9-fold higher among patients with chronic opioid prescriptions compared to those with no opioid prescriptions. Second, the liver-specific morbidity of opioids was limited to HE, and not other decompensation events. These findings suggest that opioids, particularly when used chronically, may not be safe even in younger patients with well-compensated cirrhosis.

\subsection{Opioids are uniquely unsafe in cirrhosis}

These data expand the literature on the risks of opioids in cirrhosis in multiple ways. First, using positive and negative controls, we showed that the risk of $\mathrm{HE}$ was specific to opioid exposure, decreasing concern for confounding by indication. Second, there was no association between opioid prescriptions and other decompensation events, suggesting that our results were not confounded by the severity of liver disease. In other words, if opioid use was more frequent in patients with more advanced cirrhosis, we would expect all decompensation events to be associated with opioid prescriptions. Third, we found that these associations are present even among younger patients with well-compensated cirrhosis.

Our findings add to the data from Medicare demonstrating that opioids are associated with an increased risk of HE. ${ }^{14}$ This Medicare study included a much older population than our current study and did not limit their population to those with well-compensated cirrhosis. Our findings demonstrate that this potential harm of opioids extends to younger patients with well-compensated cirrhosis.

\subsection{Implications of these findings}

In light of the association between opioids and $\mathrm{HE}$, providers should explore nonpharmacologic and non-opioid options for pain control. Exercise, including resistance training, might be a particularly attractive nonpharmacologic strategy for pain control given that it improves muscle mass and combats sarcopenia, which is associated with the development of HE and poor outcomes in those with cirrhosis. ${ }^{4,24-28}$ Topical therapies like capsaicin and low-dose acetaminophen ( $\leq 2 \mathrm{~g} /$ 
TABLE 1 Baseline patient characteristics by opioid use status

\begin{tabular}{|c|c|c|c|c|}
\hline & $\begin{array}{l}\text { No opioids } \\
(n=4645)\end{array}$ & $\begin{array}{l}\text { Short-term opioids } \\
(n=1505)\end{array}$ & $\begin{array}{l}\text { Chronic opioids } \\
(n=301)\end{array}$ & $P$-value \\
\hline $\begin{array}{l}\text { Age, y (median, } \\
\left.\mathrm{p}_{25}-\mathrm{p}_{75}\right)\end{array}$ & $54(49-58)$ & $53(48-58)$ & $53(50-58)$ & 0.092 \\
\hline Male Sex & $2786(60.0)$ & $774(51.4)$ & $175(58.1)$ & $<0.001$ \\
\hline \multicolumn{5}{|l|}{ Region } \\
\hline East & 912 (19.6) & $244(16.2)$ & $49(16.3)$ & \multirow[t]{4}{*}{0.024} \\
\hline Midwest & $1388(29.9)$ & $455(30.2)$ & $68(22.6)$ & \\
\hline South & $1372(29.5)$ & $377(25.0)$ & $60(19.9)$ & \\
\hline West & $973(20.9)$ & $429(28.5)$ & $124(41.2)$ & \\
\hline \multicolumn{5}{|l|}{$\mathrm{CCl}(\%)$} \\
\hline 1 & $2244(48.3)$ & $540(35.9)$ & 95 (31.6) & \multirow[t]{3}{*}{$<0.001$} \\
\hline 2 & $1303(28.1)$ & $465(30.9)$ & 87 (28.9) & \\
\hline$>2$ & $1098(23.6)$ & $500(33.2)$ & $119(39.5)$ & \\
\hline Depression & $508(10.9)$ & 254 (16.9) & $74(24.6)$ & $<0.001$ \\
\hline Diabetes mellitus & $1154(24.8)$ & $462(30.7)$ & 94 (31.2) & $<0.001$ \\
\hline \multicolumn{5}{|l|}{ Cirrhosis aetiology } \\
\hline ALD & 747 (16.1) & $222(14.8)$ & $27(9.0)$ & \multirow[t]{3}{*}{$<0.001$} \\
\hline Viral hepatitis & $1660(35.7)$ & $509(33.8)$ & $139(46.2)$ & \\
\hline Other & $2238(48.2)$ & $774(51.4)$ & $135(44.9)$ & \\
\hline TCA use & $224(4.8)$ & $158(10.5)$ & $47(15.6)$ & $<0.001$ \\
\hline Statin use & $392(8.4)$ & $194(12.9)$ & $32(10.6)$ & $<0.001$ \\
\hline Levothyroxine use & $92(2.0)$ & $40(2.7)$ & $9(3.0)$ & 0.183 \\
\hline Gabapentinoid use & $59(1.3)$ & $50(3.3)$ & $37(12.3)$ & $<0.001$ \\
\hline Benzodiazepine use & $498(10.7)$ & $283(18.8)$ & $111(36.9)$ & $<0.001$ \\
\hline Prior opioids ${ }^{b}$ & 701 (15.1) & $607(40.3)$ & $261(86.7)$ & $<0.001$ \\
\hline
\end{tabular}

Note: Values within parenthesis are expressed in \% and/or p25-p75.

Abbreviations: ALD, alcohol-related liver disease; $\mathrm{CCl}$, Charlson Comorbidity Index; TCA, tricyclic antidepressant.

${ }^{a}$ Bivariate comparison via Kruskal-Wallis test for continuous variables and chi-squared for categorical variables.

${ }^{\mathrm{b}}$ Opioid prescription in the year prior to cirrhosis diagnosis.

day) should be considered the first-line analgesic options for patients with cirrhosis given their low risk of harms. Additional studies are needed of other analgesics, including gabapentin, which has uncertain efficacy and potential harms, especially in patients with cirrhosis. ${ }^{13}$

The high incidence of $\mathrm{HE}$ among chronic opioid users in our cohort reinforces the importance of minimising the duration of opioid use when they are needed. This is particularly important given the uncertain effectiveness of chronic opioids for treating pain. ${ }^{29}$ When opioids are required in patients with cirrhosis, providers should maintain a high vigilance for identifying HE. Despite recommendations to screen patients with cirrhosis for covert HE (a precursor to overt HE that can be treated with lactulose or rifaximin) most patients do not receive this screening. ${ }^{30,31}$ Given our findings, providers could consider screening well-compensated cirrhosis patients on opioids for overt hepatic encephalopathy with point of care applications like the EncephalApp Stroop smartphone App or the four validated questions of the Sickness Index Profile followed by formal psychometric testing. ${ }^{32-34}$

\subsection{Study strengths and limitations}

This study has many strengths including its large, nationwide cohort of privately insured individuals. Moreover, we provided a novel examination of the potential risks of opioids among younger patients with no recent decompensation events. However, our findings must be interpreted in the context of this study's potential limitations.

First, given the lack of laboratory values in our data set, we were unable to compute model for end-stage liver disease or Child-Pugh Turcotte scores in order to account for the severity of liver disease. To mitigate this limitation, we restricted the cohort to those with well-compensated cirrhosis. Relatedly, there is the potential for confounding introduced as a result of increased opioid use among patients with more severe liver disease or other unmeasured confounders common to patients with pain. However, the use of landmark analysis, the lack of association between positive/negative exposure controls on $\mathrm{HE}$ events and the similar rates of non-HE 
TABLE 2 Association between opioids and positive/negative controls on hepatic encephalopathy

\begin{tabular}{|c|c|c|c|c|}
\hline & Number of patients (\%) & $\begin{array}{l}\text { Number who developed } \\
\text { HE/1 y (\%) }\end{array}$ & Crude HR $(95 \% \mathrm{Cl})$ & $\begin{array}{l}\text { Adjusted } \mathrm{HR}^{\mathrm{a}}(95 \% \\
\mathrm{Cl})\end{array}$ \\
\hline \multicolumn{5}{|l|}{ Opioids } \\
\hline Short-term opioids & $1505(23.3)$ & $76(5.0)$ & $1.53(1.16-2.01)$ & $1.44(1.07-1.94)$ \\
\hline Chronic opioids & $301(4.7)$ & 19 (6.3) & $1.94(1.20-3.12)$ & $1.83(1.07-3.12)$ \\
\hline No opioids or gabapentin & $4586(71.1)$ & $151(3.3)$ & Ref. & Ref. \\
\hline Short-term opioids & 1505 (23.3) & $76(5.0)$ & $1.55(1.17-2.04)$ & $1.46(1.08-1.97)$ \\
\hline Chronic opioids & $301(4.7)$ & 19 (6.3) & $1.96(1.22-3.16)$ & $1.86(1.09-3.19)$ \\
\hline Gabapentin only & $59(0.9)$ & $4(6.8)$ & $2.10(0.78-5.68)$ & $1.68(0.62-4.59)$ \\
\hline \multicolumn{5}{|l|}{ Tricyclic antidepressants } \\
\hline Chronic opioids & $301(4.7)$ & $19(6.3)$ & $1.94(1.21-3.13)$ & $1.81(1.06-3.11)$ \\
\hline Tricyclic antidepressants only & $224(3.5)$ & $8(3.6)$ & $1.08(0.53-2.20)$ & $0.90(0.44-1.86)$ \\
\hline \multicolumn{5}{|l|}{ Levothyroxine } \\
\hline No opioids or levothyroxine & $4553(70.6)$ & $151(3.3)$ & Ref. & Ref. \\
\hline Short-term opioids & $1505(23.3)$ & $76(5.0)$ & $1.53(1.17-2.03)$ & $1.45(1.08-1.96)$ \\
\hline Chronic opioids & $301(4.7)$ & $19(6.3)$ & $1.95(1.21-3.14)$ & $1.84(1.08-3.14)$ \\
\hline Levothyroxine only & $92(1.4)$ & $4(4.3)$ & $1.34(0.50-3.62)$ & $1.34(0.49-3.64)$ \\
\hline \multicolumn{5}{|l|}{ Statins } \\
\hline Short-term opioids & $1505(23.3)$ & $76(5.0)$ & $1.65(1.24-2.19)$ & $1.54(1.13-2.09)$ \\
\hline Chronic opioids & $301(4.7)$ & $19(6.3)$ & $2.09(1.29-3.39)$ & $1.95(1.14-3.35)$ \\
\hline Benzodiazepines only & $498(7.7)$ & $27(5.4)$ & $1.77(1.17-2.68)$ & $1.51(0.98-2.31)$ \\
\hline
\end{tabular}

${ }^{a}$ Adjusted for age, sex, region, cirrhosis aetiology, depression, Charlson comorbidity index (CCI) and prior opioid use.

decompensation events within opioid groups all provide reassurance against confounding.

Given that our outcome definitions relied on ICD codes and medication prescription data, there is a possibility of misclassification of $\mathrm{HE}$ cases and other decompensation events. For instance, it may be that opioid-induced altered mental status is being misdiagnosed as $\mathrm{HE}$. However, the annual incidence of $\mathrm{HE}$ and other decompensation events are comparable to what has been published in the literature, providing external validity to our results. ${ }^{15,35-38}$ In addition, any event prompting a billing code for $\mathrm{HE}$ or the initiation of lactulose/rifaximin is likely to be clinically important. Furthermore, the separation of exposure and outcome assessment through the landmark analysis limits the inclusion of episodes of altered mental status occurring immediately after opioid initiation, patients who were treated with lactulose for opioid-induced constipation or put on lactulose or rifaximin as HE prophylaxis after being started on opioids. Lastly, a 6-month exposure period was chosen a priori to reduce the risk of a type II error, confounding by indication and misclassification of opioid use. While the sensitivity analyses using exposure periods of 3 and 9 months had some nonsignificant results, the direction of these associations remained and we suspect these were due to misclassification of opioid use and type II error respectively.

\section{5 | CONCLUSION}

In this national study of younger, privately insured patients with cirrhosis and no recent decompensation events, prescriptions for opioids were associated with $\mathrm{HE}$. In light of this potential harm of opioid 
Cumulative Incidence of Hepatic Encephalopathy by Opioid Status

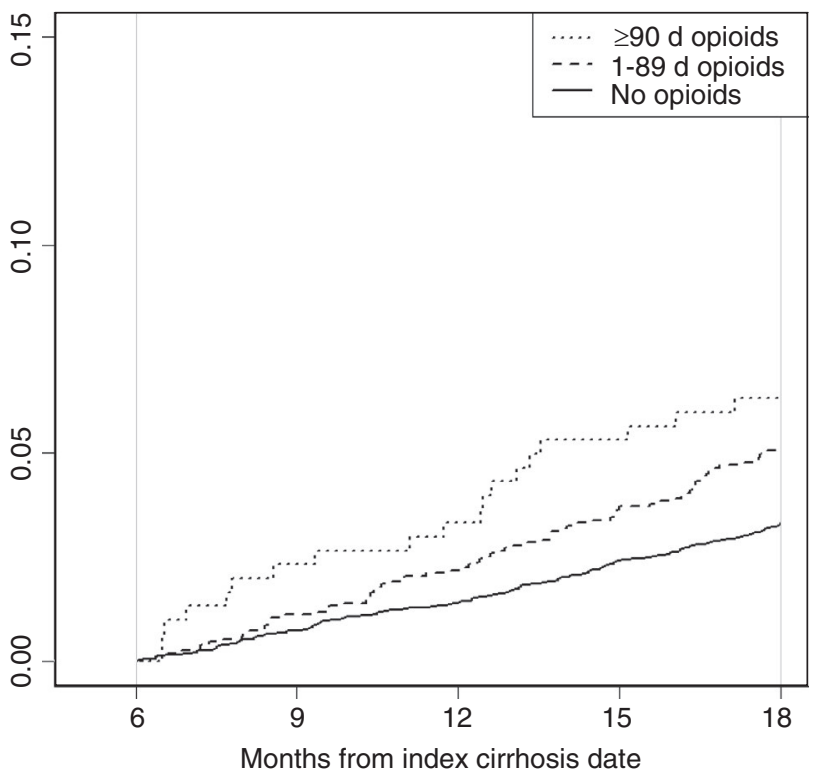

FIGURE 3 Cumulative incidence of hepatic encephalopathy among patients with previously compensated cirrhosis by opioid use. The cumulative incidence of hepatic encephalopathy over a year among patients with no opioid prescriptions, short-term opioids and chronic opioid prescriptions during our exposure assessment period (0-6 mo after cirrhosis diagnosis)

prescriptions, these medications should be avoided when possible

Cumulative Incidence of Other Events by Opioid Status

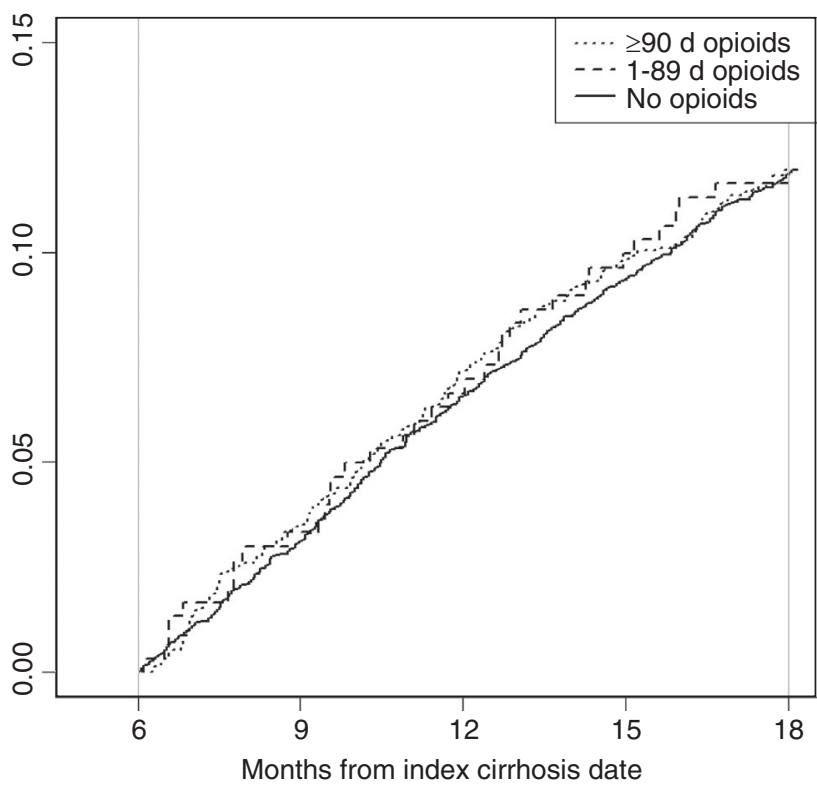

FIGURE 4 Cumulative incidence of non-HE decompensation events among patients with previously compensated cirrhosis by opioid use. The cumulative incidence of nonhepatic encephalopathy decompensation events over a year among patients with no opioids, short-term opioid prescriptions and chronic opioid prescriptions during our exposure assessment period (0-6 months after cirrhosis diagnosis) even in patients with well-compensated cirrhosis. When opioid use is required, it should be short duration and clinicians should maintain a high vigilance for detecting HE. These associations could also be further explored with prospective studies of the safety of opioids in patients with cirrhosis and additional study investigating the mechanisms linking opioids and $\mathrm{HE}$.

\section{ACKNOWLEDGEMENT}

Declaration of personal interests: Andrew M. Moon, Yue Jiang, Shari S. Rogal, Sarah R. Lieber and A. Sidney Barritt: No potential personal or financial conflict to disclose. Elliot Tapper: Received grant funding from Valeant and Gilead; consulted for Allergan and Novartis; participated in advisory boards for Bausch Health and Mallinckrodt.

\section{AUTHORSHIP}

Guarantor of the article: Andrew M. Moon.

Author contributions: Andrew M. Moon: Study concept and design, interpretation of data, drafting and critical revision of manuscript. Yue Jiang: Study concept and design, data extraction, statistical analysis, critical revision of manuscript. Shari S. Rogal, Elliot B. Tapper, Sarah R. Lieber and A. Sidney Barritt: Study concept and design, interpretation of data and critical revision of manuscript. All authors approved the final version of this manuscript.

\section{ORCID}

Andrew M. Moon (iD https://orcid.org/0000-0001-7163-2062

Elliot B. Tapper (iD https://orcid.org/0000-0002-0839-1515

\section{REFERENCES}

1. Jepsen P, Ott P, Andersen PK, Sorensen HT, Vilstrup H. Clinical course of alcoholic liver cirrhosis: a Danish population-based cohort study. Hepatology. 2010;51:1675-1682.

2. Lucidi C, Ginanni Corradini S, Abraldes JG, et al. Hepatic encephalopathy expands the predictivity of model for end-stage liver disease in liver transplant setting: evidence by means of 2 independent cohorts. Liver Transpl. 2016;22:1333-1342.

3. Bajaj JS, Wade JB, Gibson DP, et al. The multi-dimensional burden of cirrhosis and hepatic encephalopathy on patients and caregivers. Am J Gastroenterol. 2011;106:1646-1653.

4. Tapper EB, Jiang ZG, Patwardhan VR. Refining the ammonia hypothesis: a physiology-driven approach to the treatment of hepatic encephalopathy. Mayo Clin Proc. 2015;90:646-658.

5. Dorn S, Lembo A, Cremonini F. Opioid-induced bowel dysfunction: epidemiology, pathophysiology, diagnosis, and initial therapeutic approach. Am J Gastroenterol Suppl. 2014;2:31-37.

6. Acharya C, Betrapally NS, Gillevet PM, et al. Chronic opioid use is associated with altered gut microbiota and predicts readmissions in patients with cirrhosis. Aliment Pharmacol Ther. 2017;45:319-331.

7. Bergasa NV, Rothman RB, Mukerjee E, Vergalla J, Jones EA. Upregulation of central mu-opioid receptors in a model of hepatic encephalopathy: a potential mechanism for increased sensitivity to morphine in liver failure. Life Sci. 2002;70:1701-1708.

8. Rogal SS, Beste LA, Youk A, et al. Characteristics of opioid prescriptions to veterans with cirrhosis. Clin Gastroenterol Hepatol. 2019;17:1165-1174:e1163. 
9. Konerman MA, Rogers M, Kenney B, et al. Opioid and benzodiazepine prescription among patients with cirrhosis compared to other forms of chronic disease. BMJ Open Gastroenterol. 2019;6:e000271.

10. Rogal SS, Bielefeldt K, Wasan AD, et al. Inflammation, psychiatric symptoms, and opioid use are associated with pain and disability in patients with cirrhosis. Clin Gastroenterol Hepatol. 2015;13:1009-1016.

11. Rogal SS, Winger D, Bielefeldt K, Szigethy E. Pain and opioid use in chronic liver disease. Dig Dis Sci. 2013;58:2976-2985.

12. Madan A, Barth KS, Balliet WE, et al. Chronic pain among liver transplant candidates. Prog Transplant. 2012;22:379-384.

13. Chandok N, Watt KD. Pain management in the cirrhotic patient: the clinical challenge. Mayo Clin Proc. 2010;85:451-458.

14. Tapper EB, Henderson JB, Parikh ND, Ioannou GN, Lok AS. Incidence of and risk factors for hepatic encephalopathy in a population-based cohort of Americans with cirrhosis. Hepatol Commun. 2019;3:1510-1519.

15. Tapper EB, Parikh ND, Sengupta N, et al. A risk score to predict the development of hepatic encephalopathy in a population-based cohort of patients with cirrhosis. Hepatology. 2018;68:1498-1507. https://doi.org/10.1002/hep.29628.

16. Stempel DA, Mauskopf J, McLaughlin T, Yazdani C, Stanford RH. Comparison of asthma costs in patients starting fluticasone propionate compared to patients starting montelukast. Respir Med. 2001;95:227-234.

17. Kramer JR, Davila JA, Miller ED, Richardson P, Giordano TP, ElSerag HB. The validity of viral hepatitis and chronic liver disease diagnoses in Veterans Affairs administrative databases. Aliment Pharmacol Ther. 2008;27:274-282.

18. Ioannou GN, Green P, Lowy E, Mun EJ, Berry K. Differences in hepatocellular carcinoma risk, predictors and trends over time according to etiology of cirrhosis. PLoS ONE. 2018;13:e0204412.

19. Nehra MS, Ma Y, Clark C, Amarasingham R, Rockey DC, Singal AG. Use of administrative claims data for identifying patients with cirrhosis. J Clin Gastroenterol. 2013;47:e50-54.

20. Goldberg D, Lewis J, Halpern S, Weiner M, Lo Re V, III. Validation of three coding algorithms to identify patients with end-stage liver disease in an administrative database. Pharmacoepidemiol Drug Saf. 2012;21:765-769.

21. Lipsitch M, Tchetgen Tchetgen E, Cohen T. Negative controls: a tool for detecting confounding and bias in observational studies. Epidemiology. 2010;21:383-388.

22. Lund JL, Richardson DB, Sturmer T. The active comparator, new user study design in pharmacoepidemiology: historical foundations and contemporary application. Curr Epidemiol Rep. 2015;2:221-228.

23. Dafni U. Landmark analysis at the 25 -year landmark point. Circ Cardiovasc Qual Outcomes. 2011;4:363-371.

24. Aamann L, Dam G, Borre M, et al. Resistance training increases muscle strength and muscle size in patients with liver cirrhosis. Clin Gastroenterol Hepatol. 2019;:S1542-3565(19)30847-X.

25. Busch AJ, Barber KA, Overend TJ, Peloso PM, Schachter CL. Exercise for treating fibromyalgia syndrome. Cochrane Database Syst Rev. 2007;4:CD003786.

26. Fransen M, McConnell S, Hernandez-Molina G, Reichenbach S. Exercise for osteoarthritis of the hip. Cochrane Database Syst Rev. 2014;CD007912.
27. Fransen $M$, McConnell $S$, Harmer AR, Van der Esch M, Simic M, Bennell KL. Bennell KL. Exercise for osteoarthritis of the knee. Cochrane Database Syst Rev. 2015;1:CD004376.

28. Hayden JA, van Tulder MW, Malmivaara A, Koes BW. Exercise therapy for treatment of non-specific low back pain. Cochrane Database Syst Rev. 2005;3:CD000335.

29. Busse JW, Wang LI, Kamaleldin M, et al. Opioids for chronic noncancer pain: a systematic review and meta-analysis. JAMA. 2018;320:2448-2460.

30. Vilstrup H, Amodio P, Bajaj J, et al. Hepatic encephalopathy in chronic liver disease: 2014 Practice Guideline by the American Association for the Study of Liver Diseases and the European Association for the Study of the Liver. Hepatology. 2014;60:715-735.

31. Bajaj JS, Etemadian A, Hafeezullah M, Saeian K. Testing for minimal hepatic encephalopathy in the United States: an AASLD survey. Hepatology. 2007;45:833-834.

32. Bajaj JS, Heuman DM, Sterling RK, et al. Validation of EncephalApp, smartphone-based Stroop test, for the diagnosis of covert hepatic encephalopathy. Clin Gastroenterol Hepatol. 2015; 13(10):18281835.e1.

33. Nabi E, Thacker LR, Wade JB, et al. Diagnosis of covert hepatic encephalopathy without specialized tests. Clin Gastroenterol Hepatol. 2014;12:1384-1389.e2.

34. Patidar KR, Bajaj JS. Covert and overt hepatic encephalopathy: diagnosis and management. Clin Gastroenterol Hepatol. 2015;13:2048-2061.

35. Liu TL, Barritt AI, Weinberger M, Paul JE, Fried B, Trogdon JG. Who treats patients with diabetes and compensated cirrhosis. PLOS ONE. 2016;11:e0165574.

36. Tapper EB, Baki J, Parikh N, Lok AS. Incidence and predictors of hepatic encephalopathy in a population-based cohort of older Americans with cirrhosis: role of opiates, benzodiazepines and proton-pump inhibitors (PPIs). Paper presented at: The Liver Meeting 2018; San Francisco, CA.

37. D'Amico G, Garcia-Tsao G, Pagliaro L. Natural history and prognostic indicators of survival in cirrhosis: a systematic review of 118 studies. J Hepatol. 2006;44:217-231.

38. Fleming KM, Aithal GP, Card TR, West J. The rate of decompensation and clinical progression of disease in people with cirrhosis: a cohort study. Aliment Pharmacol Ther. 2010;32:1343-1350.

\section{SUPPORTING INFORMATION}

Additional supporting information will be found online in the Supporting Information section.

How to cite this article: Moon AM, Jiang Y, Rogal SS, Tapper EB, Lieber SR, Barritt AS IV. Opioid prescriptions are associated with hepatic encephalopathy in a national cohort of patients with compensated cirrhosis. Aliment Pharmacol Ther. 2020;51:652-660. https://doi.org/10.1111/apt.15639 